\title{
Limitations on the recovery of the true AGN variability parameters using damped random walk modeling
}

\author{
Szymon Kozłowski
}

\begin{abstract}
Warsaw University Observatory, Al. Ujazdowskie 4, 00-478 Warszawa, Poland e-mail: simkoz@astrouw.edu.pl
\end{abstract}

Received 13 October 2016 / Accepted 23 November 2016

\begin{abstract}
Context. The damped random walk (DRW) stochastic process is nowadays frequently used to model aperiodic light curves of active galactic nuclei (AGNs). A number of correlations between the DRW model parameters, the signal decorrelation timescale and amplitude, and the physical AGN parameters, such as the black hole mass or luminosity, have been reported.

Aims. We are interested in whether or not it is plausible to correctly measure the DRW parameters from a typical ground-based survey, and, in particular, in how accurate the recovered DRW parameters are compared to the input ones.

Methods. By means of Monte Carlo simulations of AGN light curves, we studied the impact of the light curve length, the source magnitude (the photometric properties of a survey), cadence, and additional light (e.g., from a host galaxy) on the DRW model parameters.

Results. The most significant finding is that currently existing surveys are going to return unconstrained DRW decorrelation timescales, because typical rest-frame data do not probe long enough timescales or the white noise part of the power spectral density for DRW. The experiment length must be at least ten times longer than the true DRW decorrelation timescale, being presumably in the vicinity of one year, thus meaning the necessity for AGN light curves measuring a minimum of 10 years (rest-frame). The DRW timescales for sufficiently long light curves are typically weakly biased, and the exact bias depends on the fitting method and used priors. The DRW amplitude is mostly affected by the photometric noise (the source magnitude or the signal-to-noise ratio), cadence, and the AGN host light.

Conclusions. Because the DRW parameters appear to be incorrectly determined from typically existing data, the reported correlations of the DRW variability and physical AGN parameters from other works seem unlikely to be correct. In particular, the anti-correlation of the DRW decorrelation timescale with redshift is a manifestation of the survey length being too short. Application of DRW to modeling typical AGN optical light curves is questioned.
\end{abstract}

Key words. accretion, accretion disks - quasars: general - galaxies: active - methods: data analysis

\section{Introduction}

Active galactic nuclei (AGNs) variability studies have already entered a new era. This is primarily due to a gigantic increase in data volume from the already existing and forthcoming deep, large sky surveys, but also due to introduction of new methods that enable direct modeling of aperiodic AGN light curves. They are nowadays routinely modeled using the damped random walk (DRW) model (Kelly et al. 2009; Kozłowski et al. 2010; MacLeod et al. 2010, 2011, 2012, Butler \& Bloom 2011; Ruan et al. 2012; Zu et al. 2011, 2013, 2016), with potentially some departures as seen both in steeper than DRW power spectral distributions (PSDs) of AGNs from the Kepler mission (Mushotzky et al. 2011; Kasliwal et al. 2015), steeper than DRW PSDs for more massive Pan-STARRS (Simm et al. 2016) and PTF/iPTF AGNs (Caplar et al. 2016), and from steeper than DRW structure functions (SFs) for luminous SDSS AGNs (Kozłowski 2016a). Modeling aperiodic AGN light curves that seem to have a non-DRW covariance matrix of the signal is perfectly feasible using a DRW model, however biased DRW parameters are expected (Kozłowski 2016b). Kelly et al. (2014) recently considered a broader class of continuous-time autoregressive moving average (CARMA) models of which DRW is the simplest variant.
An AGN light curve can be described (and fitted) with DRW having just two model parameters: the damping timescale $\tau$ (the signal decorrelation timescale) and the modified variability amplitude $\hat{\sigma}$ (or equivalently $\mathrm{SF}_{\infty}=\hat{\sigma} \sqrt{\tau}$; MacLeod et al. 2010). Kelly et al. (2009) reported on correlations between the DRW model parameters and physical properties of AGNs. Using approximately 9000 SDSS quasars, MacLeod et al. (2010) analyzed these correlations in detail and showed that the two model parameters are correlated with the wavelength, luminosity and/or black hole mass. While such correlations are generally expected, we will show that the reported ones are likely spurious.

This paper is inspired by the curious fact that cutting a typical, several-year-long AGN light curve in shorter fragments, changes the measured DRW parameters while the true underlying stochastic process does not change. The importance of the light curve length for DRW was briefly mentioned in MacLeod et al. (2010) and Kozłowski (2016a) (and recently recognized in Fausnaugh et al. 2016), but, as we show here, is significantly underestimated (or not well understood). In this paper, we thoroughly study all plausible secondary effects on the measured DRW parameters and show that DRW is not a simple "black box" that returns correct parameters. In particular, we are interested in how well we are able to estimate the DRW parameters from the ground-based light curves, given a range of ratios 

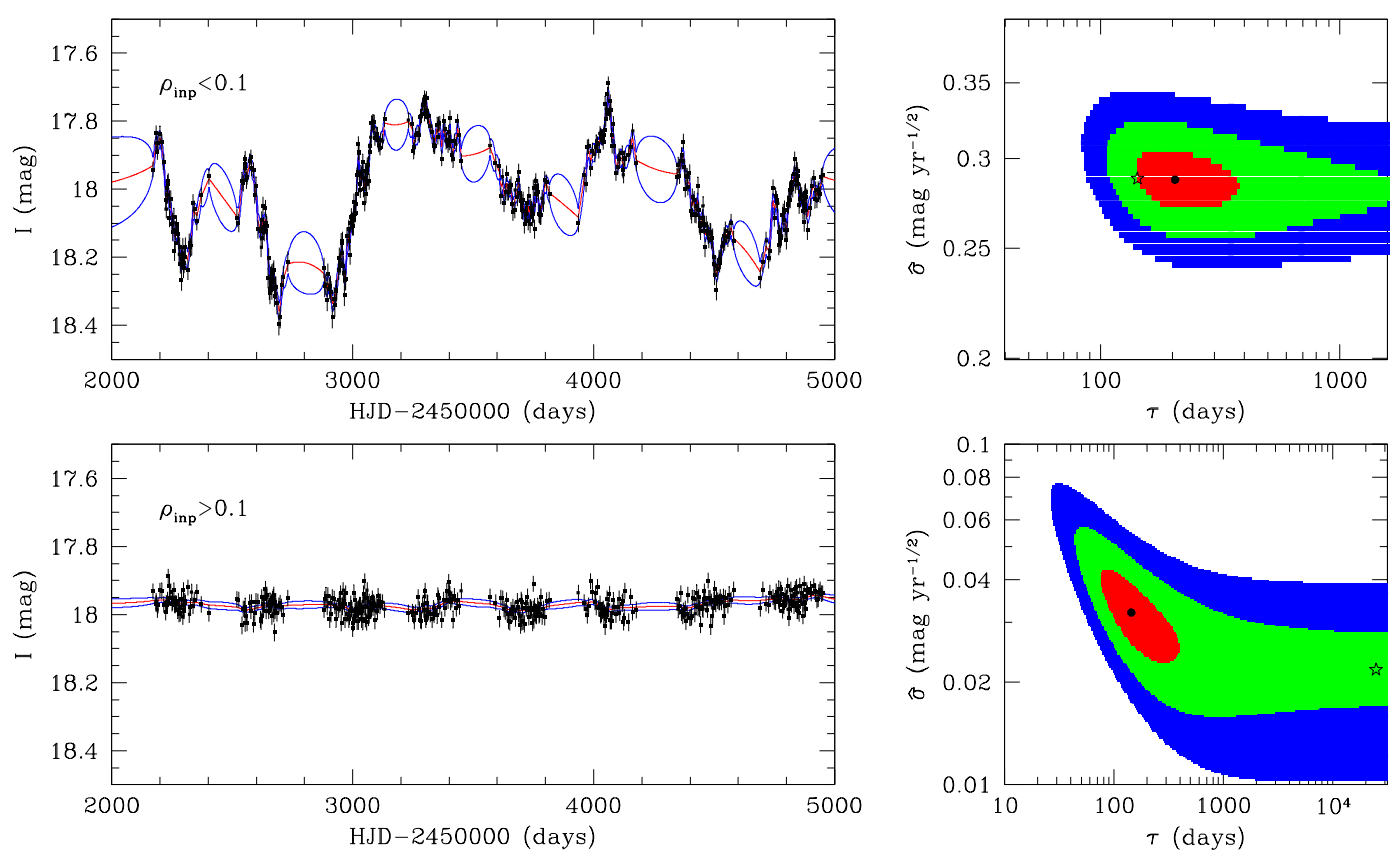

Fig. 1. Left column: two examples of simulated AGN light curves for OGLE-III. Both light curves have the mean magnitude $I=18$ mag, $\mathrm{SF}_{\infty}=0.20 \mathrm{mag}$, but the input timescale is $\tau_{\text {inp }}=150 \mathrm{~d}$ (top) and $25000 \mathrm{~d}$ (bottom). The experiment lasted approximately $t_{\exp }=2800 \mathrm{~d}$. Thus, the ratio of the decorrelation timescale to the experiment length $\rho_{\text {inp }}=\tau_{\text {inp }} t_{\text {exp }}^{-1}$ is 0.05 and 9.0, respectively. The red line is the best-fit DRW model and the blue lines present $1 \sigma$ "error snakes". Right column: model likelihood surface for the two light curves. The black point is the maximum likelihood point, while the red, green, and blue areas represent $\Delta \ln \mathcal{L}=-0.5,-2.0$, and -4.5 , corresponding to $\Delta \chi^{2}=1,4$, and 9 , (or 1,2 , and $3 \sigma$ ), respectively. The open star marks the input parameters. The bias in the measured parameters is obvious in both cases (dots and stars do not match).

( $\left.\rho_{\text {inp }}=\tau_{\text {inp }} t_{\text {exp }}^{-1}\right)$ of the input decorrelation timescale $\left(\tau_{\text {inp }}\right)$ to the experiment length $\left(t_{\text {exp }}\right)$, different cadences (2-80 d), light curve lengths (including four-month-long seasonal gaps), and photometric properties of a typical ground-based survey (here the Optical Gravitational Lensing Experiment ${ }^{1}$ (OGLE) and/or the Sloan Digital Sky Survey ${ }^{2}$ (SDSS)). We will perform extensive Monte Carlo simulations and modeling of AGN light curves using DRW to study the biases between the input (hence, assumed to be true) and output (measured) model parameters as a function of the source and survey properties. In Sect. 2, we present the simulation and modeling setup, while the results are discussed in Sect. 3. The paper is summarized in Sect. 4.

\section{DRW simulations and modeling}

We simulate and then model AGN light curves as a DRW stochastic process that is characterized by the covariance matrix of the signal

$S_{i j}=\sigma^{2} \exp \left(-\frac{\left|t_{i}-t_{j}\right|}{\tau}\right)$

where $t_{i}-t_{j}=\Delta t$ is the time interval between the $i$ th and $j$ th epochs, and $\sigma^{2}$ is the signal variance, while its relation to $\hat{\sigma}$ or $\mathrm{SF}_{\infty}$ is $\sigma=\hat{\sigma} \sqrt{\tau / 2}$ and $\sigma=\mathrm{SF}_{\infty} / \sqrt{2}$ (see Kelly et al. 2009; Kozłowski et al. 2010; MacLeod et al. 2010, 2011, 2012; Butler \& Bloom 2011; Zu et al. 2011, 2013, 2016).

\footnotetext{
1 http://ogle.astrouw.edu.pl, see Udalski et al. (2015) for details.

2 http: //www. sdss.org, see York et al. (2000) for details.
}

\section{1. light curve simulations}

We generate 100-years-long light curves with a cadence of two days. They are later cut into shorter time baselines and/or have the cadence reduced, that is, degraded to the ground-based surveys considered here: SDSS Stripe 82, spanning eight years with a median of 60 epochs in the $r$-band, and the third phase of OGLE (OGLE-III), also spanning eight years with a median of 445 I-band epochs in the Large Magellanic Cloud (Fig. 1). We also consider 20-years-long OGLE light curves, where we extrapolate the existing cadence into the future.

To generate a light curve, the signal chain begins with $s_{1}=$ $G\left(\sigma^{2}\right)$, where $G\left(\sigma^{2}\right)$ is a Gaussian deviate of dispersion $\sigma$. The subsequent light curve points are recursively obtained from

$s_{i+1}=s_{i} \mathrm{e}^{-\Delta t / \tau}+G\left[\sigma^{2}\left(1-\mathrm{e}^{-2 \Delta t / \tau}\right)\right]$,

where $\Delta t=t_{i+1}-t_{i}$. The observed light curve is obtained from $y_{i}=s_{i}+G\left(n_{i}^{2}\right)+\langle y\rangle$, where $n_{i}$ is the observational photometric noise, and $\langle y\rangle$ is the mean magnitude (see also Kozłowski et al. 2010; Zu et al. 2011; Kozłowski 2016b).

One of the most important quantities is a survey's photometric noise and we adopt it here to be $\sigma_{\mathrm{OGLE}}=$ $\sqrt{0.004^{2}+\exp (1.63 \times(I-22.55))} \mathrm{mag}$ for OGLE, estimated for one of the Large Magellanic Cloud fields (Udalski et al. 2008, 2015; Skowron et al. 2016), while for the SDSS we adopt $\sigma_{\text {SDSS }}=\sqrt{0.013^{2}+\exp (2.0 \times(r-23.36))}$ mag (Ivezić et al. 2007; Kozłowski 2016a), obtained from Stripe 82 AGNs. The final simulations' cadence is akin to the original surveys. The exact choice of the input timescales and amplitudes is described in Sect. 3. 


\subsection{Light curve modeling}

The light curves are modeled by means of a method that optimally reconstructs irregularly sampled data, developed by Press et al. (1992a,b) and Rybicki \& Press (1995) (commonly referred to as the PRH method), and later adopted in AGN variability studies (Kozłowski et al. 2010; Zu et al. 2011, 2013, 2016).

While the full derivation of the method is presented in Kozłowski et al. (2010) and Zu et al. (2011, 2013, 2016), we briefly present its basic concepts here. An AGN light curve $\boldsymbol{y}(t)$ can be represented by a sum of the true variable AGN signal $s(t)$ (with the covariance matrix $\boldsymbol{S}$ ), noise $\boldsymbol{n}$ (with the covariance matrix $\boldsymbol{N}$ ), matrix $\boldsymbol{L}$, and a set of linear coefficients $\boldsymbol{q}$ (e.g., that can be used to subtract or add the mean light curve magnitude or any trends), so that $\boldsymbol{y}(t)=\boldsymbol{s}(t)+\boldsymbol{n}+\boldsymbol{L} \boldsymbol{q}$.

The likelihood of the data given $s(t), \boldsymbol{q}$, and model parameters $\tau$ and $\hat{\sigma}$ is (Eq. (A.8) in Kozłowski et al. 2010; Eq. (11) in $\mathrm{Zu}$ et al. 2013)

$\mathcal{L}(\boldsymbol{y} \mid \boldsymbol{s}, \boldsymbol{q}, \tau, \hat{\sigma})=|\boldsymbol{C}|^{-1 / 2}\left|\boldsymbol{L}^{T} \boldsymbol{C}^{-1} \boldsymbol{L}\right|^{-1 / 2} \exp \left(-\frac{\boldsymbol{y}^{T} \boldsymbol{C}_{\perp}^{-1} \boldsymbol{y}}{2}\right)$,

where $\boldsymbol{C}=\boldsymbol{S}+\boldsymbol{N}$ is the total covariance matrix of the data, and $\boldsymbol{C}_{\perp}^{-1}=\boldsymbol{C}^{-1}-\boldsymbol{C}^{-1} \boldsymbol{L}\left(\boldsymbol{L}^{T} \boldsymbol{C}^{-1} \boldsymbol{L}\right)^{-1} \boldsymbol{L}^{T} \boldsymbol{C}^{-1}$. To measure the model parameters, the likelihood $\mathcal{L}$ is optimized (maximized) in $\sim O\left(N_{\text {data }}\right)$ operations by noting that the inverse of the covariance matrix has a tridiagonal form (Rybicki \& Press 1995).

In Kozłowski et al. (2010) and MacLeod et al. (2010), the reported parameters are "best-fit values" taken at the likelihood maximum $\left(\mathcal{L}_{\text {best }}\right)$, and the $1 \sigma$ uncertainties are obtained after projecting the likelihood surface onto $1 \mathrm{D}$ for the parameter of interest and taking $\Delta \ln \left(\mathcal{L}_{\text {best }}\right)-0.5$, corresponding to $\Delta \chi^{2}=1$ (right column of Fig. 1). There are two important limits of the DRW model, when $\tau \rightarrow 0$ and $\tau \rightarrow \infty$. The former corresponds to a broadening of the photometric error bars, because the covariance matrix of the signal becomes $\sigma^{2} \delta_{i j}$. We calculate the likelihood for $\tau \rightarrow 0$, hereafter referred to as $\mathcal{L}_{\text {noise }}$, and require the best model to return a better fit by $\Delta \chi^{2}>4$, so $\ln \mathcal{L}_{\text {best }}>\ln \mathcal{L}_{\text {noise }}+2.0$. To avoid unconstrained parameters, Kozłowski et al. (2010) and MacLeod et al. (2010) used priors on the likelihood of the model parameters $P(\tau)=1 / \tau$ and $P(\hat{\sigma})=1 / \hat{\sigma}$, and we use the exact same priors throughout this manuscript.

In the PYTHON implementation of this method - the JAVELIN $^{3}$ software (Zu et al. 2011, 2013, 2016), the best solution is optimized with the AOMEBA method and the likelihood maximum is explored with MCMC to find the parameter uncertainties. One can choose to use or waive the priors on the model parameters.

\section{Discussion}

Of the two DRW model parameters, the amplitude and decorrelation timescale, the latter is of the highest interest as it can be directly linked to (or interpreted as) one of the characteristic accretion disk timescales: dynamical, thermal, or viscous (e.g., Czerny 2006; King 2008). For a typical black hole mass of $10^{8} M_{\odot}$ and the accretion thin disc radius of $0.01 \mathrm{pc}$ (with $\alpha=0.1$ ), the dynamical timescale is approximately 1 year, the thermal timescale is approximately 10 years, and the viscous timescale is of order of 1 million years (e.g., Collier \& Peterson 2001). Evidently, the first two timescales are the primary candidates for identification with the DRW variability timescales.

\footnotetext{
https://bitbucket.org/nye17/javelin
}

We begin our simulations and discussion by questioning whether or not the DRW model is able to return the input parameters in an ideal situation. We simulate light curves that are extremely long (20000 d) with 2000 data points, the decorrelation timescale is $200 \mathrm{~d}$ (many times shorter than the experiment length) and $\mathrm{SF}_{\infty}=0.20 \mathrm{mag}$. The answer is yes. The likelihood surfaces have narrow peaks and the highest likelihoods are wellmatched to the input parameters.

The next step is to study the impact of the experiment span on the recovered decorrelation timescales. To address this issue, we simulate two sets of 10000 AGN light curves with $\mathrm{SF}_{\infty}=0.20 \mathrm{mag}$, one for SDSS ( $r=17 \mathrm{mag} ; 60$ epochs; 8 years) and another for OGLE ( $I=18$ mag; 445 epochs; 8 years $)$, where the ratio $\left(\rho_{\text {inp }}=\tau_{\text {inp }} t_{\text {exp }}^{-1}\right)$ of the input DRW timescale ( $\left.\tau_{\text {inp }}\right)$ to the experiment length $\left(t_{\text {exp }}\right)$ is $0.001 \lesssim \rho_{\text {inp }} \lesssim 15$. We model these light curves with DRW and find that for output ratios $\rho_{\text {out }} \geqslant 0.1$ (so $\rho_{\text {inp }} \geqslant 0.1$ ), where $\rho_{\text {out }}=\tau_{\text {out }} t_{\text {exp }}^{-1}$, DRW returns incorrect timescales ( $\tau_{\text {out }}$; the "unconstrained" region in Fig. 2 ), while for $\rho_{\text {out }} \leqslant 0.1$ (the "biased" region) the input and output parameters are closely matched. The name of the "unconstrained" region originates from the fact that regardless of the input timescale, the output timescales have a median of approximately 20-30\% of the experiment length ( $\rho_{\text {out }} \approx 0.2-0.3$; discussed below). Reconstruction of the original timescales for AGNs from this region is impossible and we present the reason in Fig. 3: we mark the frequencies $\tau^{-1},(3 \tau)^{-1}$ and $(10 \tau)^{-1}$ on top of the power spectral density (PSD) for the DRW model. From this figure one can readily see why an experiment length shorter than $\sim 10 \tau$ is insufficient to measure $\tau$. Such light curves simply do not, or weakly, probe the flat white noise part, which is vital to the DRW timescale determination. A successful survey for AGN variability should optimally probe the PSD at both low frequencies (long timescales), that is, the white noise part, as well as all the high frequencies (short timescales) to constrain the red noise part of PSD.

As a verification, we have checked the dependence of the transition between the biased and unconstrained regions on the variability amplitude and source magnitude. We performed additional, nearly identical sets of simulations with $\mathrm{SF}_{\infty}=0.10 \mathrm{mag}$ and $\mathrm{SF}_{\infty}=0.30 \mathrm{mag}$, and $I=16-19 \mathrm{mag}$. The border is an invariant.

\subsection{The unconstrained region}

We will now consider the two regions separately, starting with the unconstrained region. This region is of particular interest because typical AGN light curves have lengths of the order of the decorrelation timescale, hence they will most likely reside in this region. Kozłowski (2016a), using a set of 9000 SDSS $r$-band AGN light curves (Ivezić et al. 2007; MacLeod et al. 2010), showed that SFs (being model independent quantities) flatten at long timescales, and the rest-frame (ensemble) decorrelation timescale is of approximately one year. We will now adopt this value for our discussion, regardless of its possible correlations with the physical AGN parameters.

The first obvious finding, as explained in the previous section, is that rest-frame light curves shorter than $\sim 10 \tau$ $\left(\rho_{\text {inp }}>0.1\right)$ will provide unreliable and therefore meaningless DRW timescales ( $\rho_{\text {out }}$ in Fig. 2$)$. AGNs are typically distant sources and have considerable redshifts $z$. The higher the AGN redshift, the shorter timescales we probe, only aggravating the situation, because the experiment length must be even greater, that is, $10 \tau(1+z)$ years or more. 

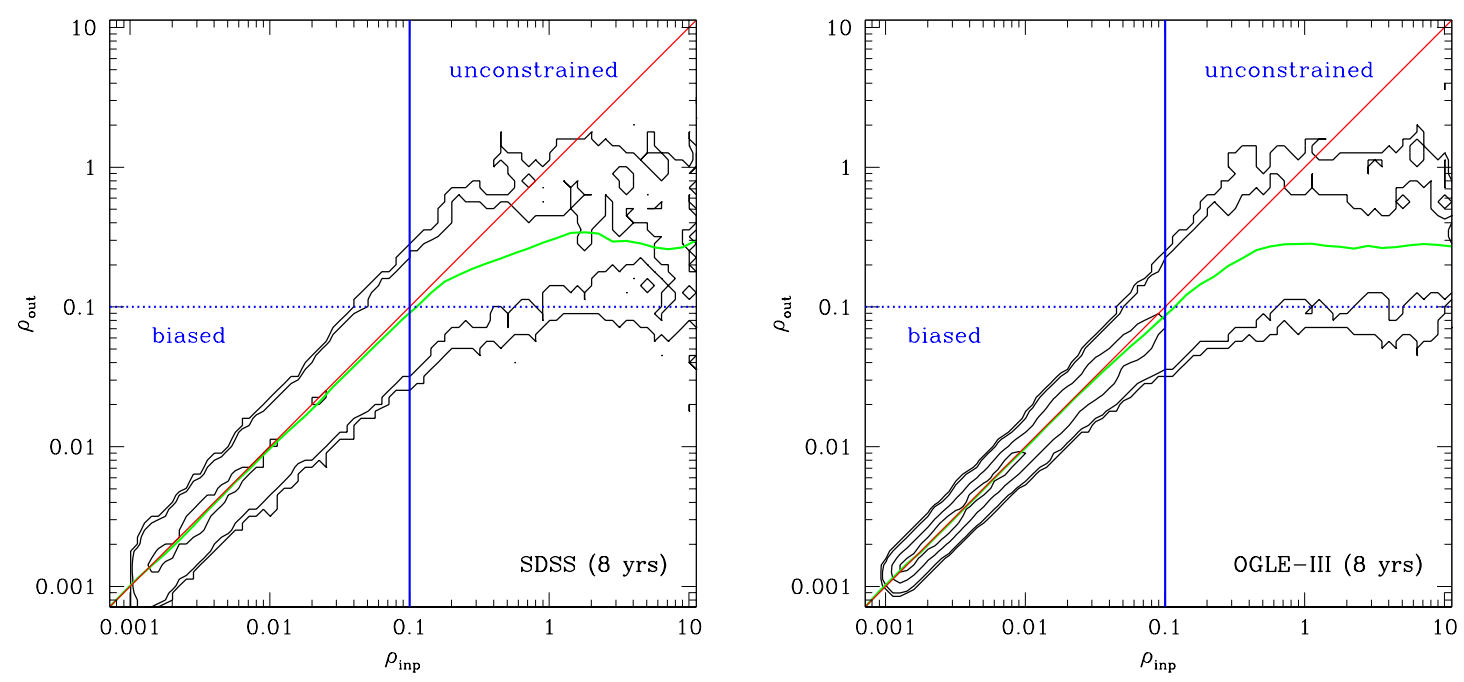

Fig. 2. Bias in the DRW decorrelation timescale caused by the experiment length. As contours we present two sets of 10000 AGN light curve realizations modeled as DRW, SDSS (60 epochs; left) and OGLE-III (445 epochs; right), with $\mathrm{SF}_{\infty}=0.20$ mag and a wide range of input timescales, where $r \approx 17 \mathrm{mag}$ and $I \approx 18 \mathrm{mag}$, respectively. AGN light curves that are long compared to the true decorrelation timescale, at least ten times longer $\left(\rho_{\text {inp }}<0.1\right)$, are the best source of the correctly measured decorrelation timescales (the "biased" region). Light curves that are short and comparable to the decorrelation timescale $\left(\rho_{\text {inp }} \approx 1\right)$ will not yield reliable decorrelation timescales and they are unconstrained (the "unconstrained" region). The typical measured timescale in the "unconstrained" region is $\sim 20-30 \%$ of the experiment span, independent of the input (true) value. The border between the biased and unconstrained regions is $\rho_{\text {inp }}=0.1$. Location of the border line is independent of the input amplitude $\mathrm{SF}_{\infty}$ and the source magnitude. While not shown, the typical uncertainty on $\rho_{\text {out }}$ is half of the outer contours.

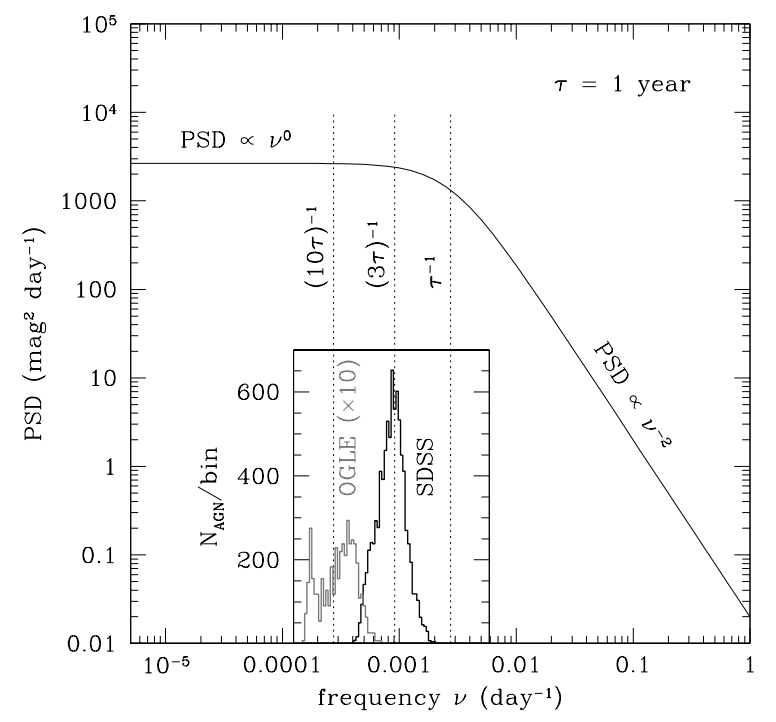

Fig. 3. Power spectral density (PSD) for DRW. We mark the frequencies $\tau^{-1},(3 \tau)^{-1}$, and $(10 \tau)^{-1}$. The experiment length shorter than $\sim 10 \tau$ is insufficient to measure $\tau$ because such light curves simply do not (for $v>\tau^{-1}$ ) or weakly (for $v>(10 \tau)^{-1}$ ) probe the white noise part (PSD $\propto$ $\left.v^{0}\right)$ of the spectrum. The inset presents the histogram of the SDSS Stripe 82 experiment lengths (hence the longest timescales probed) divided by $(1+z)$ for $\sim 9000$ SDSS AGNs and $500 I<19$ mag 20-years-long AGN light curves from combined phases of OGLE. If the true decorrelation timescale is one year then the SDSS S82 data do not probe the white noise part of PSD, so cannot constrain the decorrelation timescales.

The DRW timescale from the unconstrained region is a constant fraction of the experiment length ( $\rho_{\text {out }}$ from the unconstrained region in Fig. 2). The rest-frame experiment length is proportional to $(1+z)^{-1}$, as is the DRW timescale. From Table 1 in MacLeod et al. (2010) we learn that $\tau \propto(1+z)^{-0.7 \pm 0.5}$, which is an obvious manifestation of the experiment length effect, consistent at $0.6 \sigma$ with our interpretation that the data are too short, but also less strongly consistent with no dependence on redshift (at $1.4 \sigma$ ).

We will now investigate the shape of the distribution of the recovered decorrelation timescales from the "unconstrained" region. Figure 4 addresses this matter graphically. We consider several input timescale distributions for the SDSS survey: flat, increasing and decreasing number of AGNs per timescale bin. The recovered distributions of timescales are distant from the input ones, but they closely resemble one another. In other words, from the recovered distribution, we are unable to guess what the input distribution was. It could have been flat or have had other shapes. It is also unclear what the highest ratio could have been, because continuous distributions of ratios as high as 10 or 100, deliver almost identical final distributions.

The most notable finding is made when comparing these recovered SDSS distributions to the real SDSS timescale distribution. They are almost identical for the timescales longer than 100 days and a two-sample Kolmogorov-Smirnov (KS) test returns the significance level $P=0.67$ for the null hypothesis that the data sets are drawn from the same distribution for 81 points in both distributions (for the top left panel in Fig. 4). The bottom line here is that the true SDSS timescale distribution is unknown and it could have been flat, for example. The unconstrained SDSS timescales have been used to search for their correlations with the physical AGN parameters such as the black hole mass, luminosity, Eddington ratio, or wavelength. As shown in Fig. 4, the posterior timescale distribution has nothing to do with the input one, hence it is truly meaningless, and so are the reported correlations.

We provide a "rule of thumb" for identification of projects having AGN light curves, and hence the DRW parameters inside the "unconstrained" region. The median recovered timescale from this region is approximately $20-30 \%$ of the experiment span, regardless of the true timescale. Thus, for example, the SDSS Stripe 82 experiment lasted 2920 d (eight years) and it would be unable to correctly identify timescales longer than $\sim 300 \mathrm{~d}$ or 0.8 year at $z=0$ (greater than $10 \%$ of the experiment 

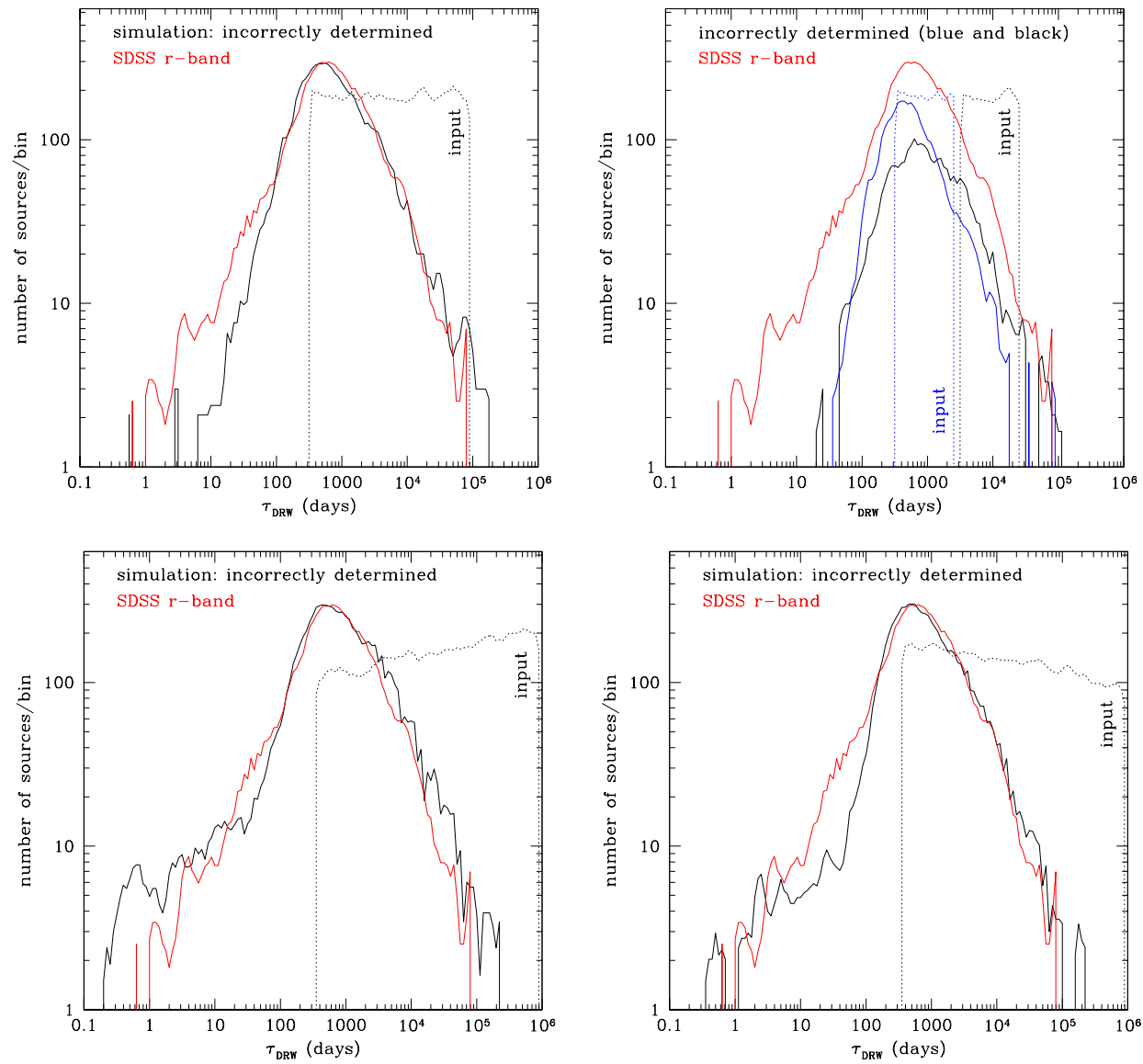

Fig. 4. Simulations and modeling of 10000 SDSS AGN light curves using DRW, where the input timescale is longer than $10 \%$ of the experiment span (from the "unconstrained" region in Fig. 2). The input distribution of $\tau$ is marked with dotted lines, while the output (measured) distribution of timescales is marked with the solid black/blue lines. The measured distribution of timescales for the real SDSS $r$-band AGN light curves is shown in red. It is obvious that DRW is unable to determine true decorrelation timescales if the input timescale is longer than $10 \%$ of the experiment span (if it belongs to the "unconstrained" region in Fig. 2). A flat input $\tau$ distribution is shown in the top left panel and is split into two flat distributions in the top right panel. In the bottom row, we used input distributions that are rising (left) and falling (right) with increasing $\tau$. All the recovered distributions look akin to the SDSS one, and for the measured timescales longer than 100 days the KS test identifies the recovered and SDSS $\tau$ distributions as drawn from the same distribution. This simply means that the timescales obtained from SDSS (and in the future Gaia/LSST/Pan-STARRS) are unconstrained and should not be used to search for correlations with the physical parameters of AGNs, because the true values of $\tau$ and hence their distribution remains unknown.

length). They will typically have the measured value of $600 \lesssim$ $\tau_{\text {out }} \lesssim 900$ days (20-30\% of the experiment length). In fact, the SDSS distribution peaks at $560 \mathrm{~d}$ and the median is $617 \mathrm{~d}$. This clearly confirms the impossibility of obtaining correct DRW timescales from SDSS. Unfortunately, it appears that the same will be the case for most past, currently existing, or near-future surveys that are rarely planned to last a decade or longer. There is clearly a possibility for correctly measuring the decorrelation timescale from the OGLE survey. There are approximately 800 AGNs observed since 2001 (16-years-long as of now) and a smaller subsample of AGNs observed since 1997 (20-yearslong; see inset of Fig. 3). If the true decorrelation timescale is indeed of approximately one year, this means that, for AGNs with $z<0.6$, the rest-frame light curves lengths will be $\sim 10$ years. There exist 115 AGNs with at least 16-years-long light curves, $z<0.6$, and $I<19.5 \mathrm{mag}$ (the photometric noise $40 \%$ of the expected AGN variability), discovered mostly (102) by the Magellanic Quasars Survey (Kozłowski et al. 2011, 2012, 2013) and a smaller number (13) from other works (Dobrzycki et al. 2002, 2003a,b, 2005; Geha et al. 2003).

Kozłowski (2016a) using optical SFs shows that $\tau \propto M^{0.4}$ (much weaker dependence than $\tau \propto M^{2.2}$ from McHardy et al. 2006 from X-rays), meaning that less massive black holes will have timescales of several months only. It is then plausible to derive meaningful DRW parameters for low redshift, low-mass black holes from surveys of approximately ten years in length, provided the cadence is sufficient, that is, the observing cadence is better than the DRW timescale. These objects will appear in the "biased" region of Fig. 2.

\subsection{The "biased" region and dependencies on other variables}

We are now interested in reconstructing the DRW parameters as a function of several survey's variables, including the average light curve magnitude, length, sampling (cadence), and additional host light for AGNs from the "biased" region of Fig. 2.

Similarly to the analysis of the unconstrained region, we perform Monte Carlo simulations of AGN light curves for the asymptotic amplitude $\mathrm{SF}_{\infty}=0.20 \mathrm{mag}$ and the decorrelation timescale of $\tau=1$ year.

From Fig. 2, we see that the typical measured timescale asymptotically approaches the true value with the increasing experiment length (with decreasing $\rho$ ).

In a large simulation set, probing cadences of 2-80 $\mathrm{d}$ and a wide magnitude range, we study the impact of data sampling 

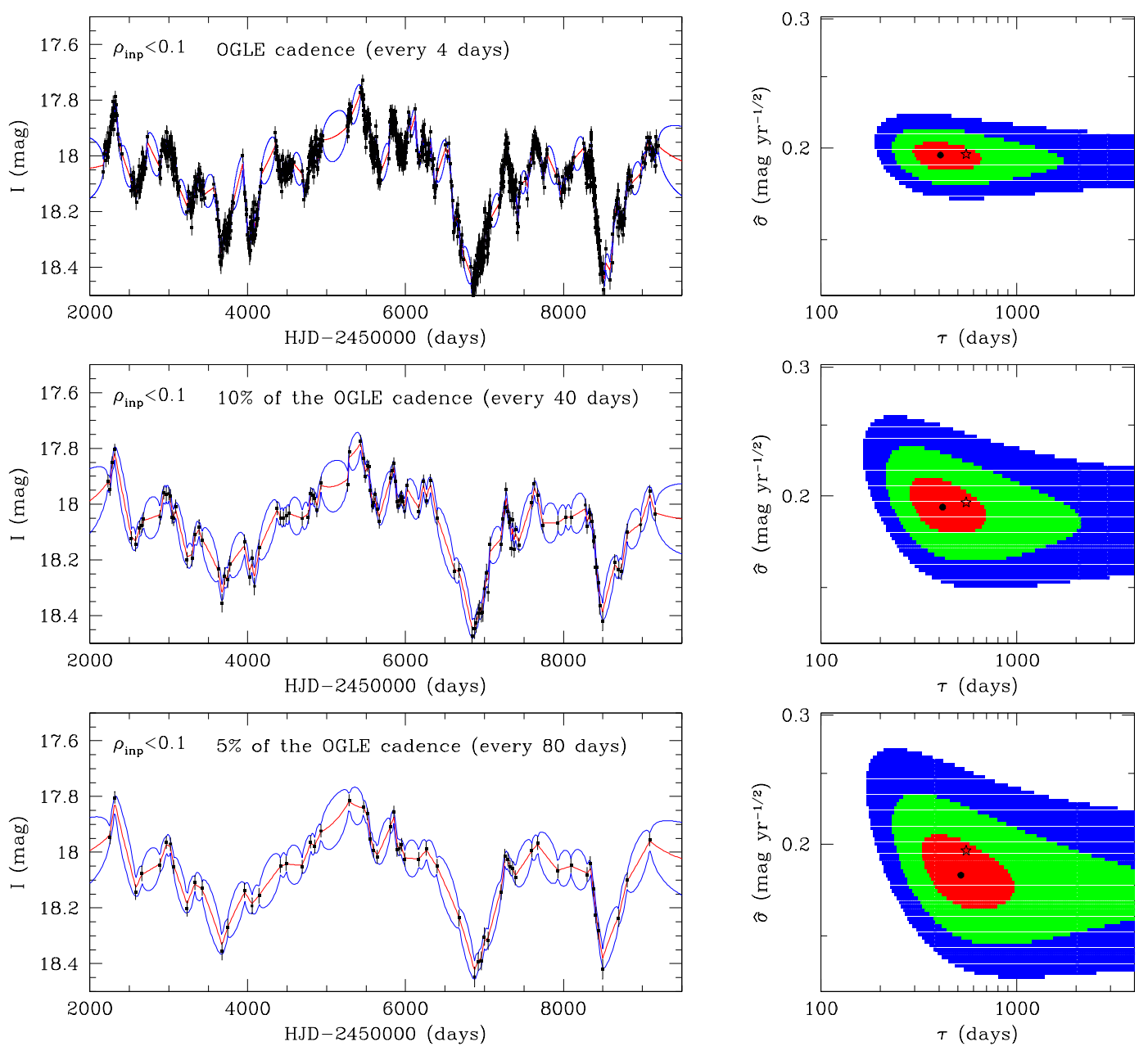

Fig. 5. Left column: simulated OGLE AGN light curves spanning 20 years, where we extrapolated the cadence into the future (top). We assumed the AGN to be at $z=0.5$ and $\tau=1$ year, so $\rho_{\text {inp }}=0.07$ (smaller than the limiting $\rho_{\text {inp }}=0.1$ ). The typical OGLE cadence during observing seasons of LMC is approximately four days. In the middle and bottom panels, we keep only every 10th and 20th point from the upper light curve. The red line is the best-fit DRW model and the blue lines present $1 \sigma$ "error snakes". The right column presents the model likelihood surface for the three light curves. The black point is the maximum likelihood point, while the red, green, and blue areas represent $\Delta \ln \mathcal{L}=-0.5,-2.0$, and -4.5 , corresponding to $\Delta \chi^{2}=1,4$, and 9 (or 1,2, and $3 \sigma$ ), respectively. The open star marks the input parameters. In all three cases, the timescale is measured with similar precision and bias, while the uncertainty in the variability amplitude increases because the middle and bottom light curves do not probe the short timescales.

on the derived DRW parameters. We find that there is almost no influence of cadence on the timescale, provided that the sampling is still better than the decorrelation timescale. In Fig. 5, we present a visualization for three example cases, where we extrapolate OGLE sampling into the future to obtain light curves of 20 years in length. We then degrade the cadence to $10 \%$ and $5 \%$ of the original and assumed an AGN to be at $z=0.5$, hence $\tau_{\text {inp }}=1(1+z)$ year, which gives $\rho_{\text {inp }} \approx 0.07$. It is obvious that the parameter uncertainties (right column of Fig. 5) are similar for the timescale (almost independent of cadence), while the amplitude uncertainty increases for sparser samplings. This is because there is less information on the high frequencies in PSD than in the original light curve. The bottom line is that we observe minute dependence of the survey's cadence on the measured timescale for $\rho_{\text {inp }}<0.1$.

An AGN light curve may contain not only the AGN light, but also additional light coming either from the AGN's host galaxy or other objects near and/or along the line-of-sight. We define blending of $20 \%$ if $80 \%$ of the light comes from an AGN and 20\% from another source. We perform 1000 Monte Carlo simulations for blending spanning $0-100 \%$ for several magnitude levels. Blending seems to weakly affect the recovered timescales, while the modified amplitude is (unsurprisingly) strongly affected by blending. The ratio of the recovered-to-input variability amplitude is directly proportional to the ratio of the AGN-to-the-total light. The goodness of fit seems to weakly improve for the increasing blending, most likely because it is easier to fit a diluted variability (or lack of it) than a full amplitude variability. The problem of AGN host light may be alleviated by using differential flux light curves.

With the increasing source magnitude (the increasing photometric noise) the ability of DRW to recover correct parameters decreases, because the modeling enters into a regime of $\ln \mathcal{L}_{\text {best }} \approx \ln \mathcal{L}_{\text {noise. }}$ In the biased region, the correct DRW timescale is recovered only when the DRW amplitude is greater than the photometric noise $\left(\sigma_{\text {DRW }}>\sigma_{\text {phot }}\right)$.

\section{Summary}

The simple procedure of cutting a typical AGN light curve in half and then modeling both the full and the two short curves with DRW has returned different variability parameters. 
This worrisome discovery has triggered us to study the impact of the experiment length, cadence, and photometric quality on the returned DRW model parameters.

The most important finding here is that to correctly measure the DRW timescale (the only parameter that can be directly linked to AGN physics), the experiment length must be at least ten times longer than the true signal decorrelation timescale (rest-frame). Otherwise, DRW is unable to correctly recognize the timescale, simply because the light curves do not probe the pink and white noise parts of PSD. We provide a rule of thumb for identification of too short AGN light curves to be modeled by DRW. If the typical measured decorrelation timescale is $\sim 20-30 \%$ of the experiment length (or more) one can be sure the timescale is measured incorrectly. This is also the case for SDSS, so the validity of correlations between DRW variability parameters and the physical AGN parameters from MacLeod et al. (2010) is refuted. In particular, these authors find that $\tau \propto(1+z)^{-0.7 \pm 0.5}$, which is an obvious manifestation of the experiment length effect on the DRW parameters, and we showed the expected correlation for data sets that are too short to use DRW is $\tau \propto(1+z)^{-1}$. At present, the capability of DRW to provide AGN variability parameters is questioned (we also note that we used priors on the likelihood of the DRW parameters $(1 / \tau$ and $1 / \hat{\sigma})$ that prevent $\tau \rightarrow \infty)$ with two exceptions: (1) because DRW is based on a method that optimally reconstructs "missing" data, it still has a lot of potential in interpolating AGN light curves. One has to guess, however, what the correct DRW parameters are for such an interpolation, and they can be obtained from SFs (Kozłowski 2016a). (2) Another avenue for measuring the DRW parameters is to analyze both low redshift and the least massive AGNs, as Kozłowski (2016a), using optical SFs, shows that $\tau \propto M^{0.4}$, meaning that at the lightweight end of the $\mathrm{BH}$ mass spectrum, the timescales should be of several months, and therefore measurable from currently existing surveys.

Most past, existing, and near-future surveys, unfortunately, are/will be unable to measure DRW timescales for the majority of AGNs, unless they span approximately two-three decades or longer, even with a sparse sampling. The first credible decorrelation timescales for individual AGNs may come from the OGLE survey, being 16 years long for 800 AGNs (or 20 years long for a small subsample) and continuously growing.

It seems that, given their planned lengths, none of the surveys, such as Gaia, LSST, or Pan-STARRS will be of sufficient length to correctly measure the decorrelation timescales for typical AGNs. They will, however, be able to probe the covariance matrix of the signal $S_{i j}$ which can be obtained from SF, as $\operatorname{SF}\left(t_{i}-t_{j}\right)=\sqrt{2 \sigma_{\mathrm{n}}^{2}+2 \sigma_{\mathrm{s}}^{2}-2 S_{i j}}$, where $\sigma_{\mathrm{s}}^{2}$ and $\sigma_{\mathrm{n}}^{2}$ are the signal and noise variances, respectively (see Kozłowski 2016a, for a review). Given a large number of AGNs monitored, these surveys will be able to trace any plausible correlations of the covariance matrix shape (via the SF slope) with the luminosity, black hole mass, and Eddington ratio.

An intriguing finding we made was that for sufficiently long AGN light curves ( $>10$ years rest-frame), the cadence of a survey seems nearly unimportant for determination of the DRW decorrelation timescale of $\tau=1$ year (at least in a considered range of 2-80 d) but the light curve sampling must still be shorter than the timescale (but high cadence is not necessary). The important lesson is that a light curve should optimally probe PSD at both red, pink, and white noise parts.
Another DRW bias was found recently by Kozłowski (2016b). He found a degeneracy in DRW modeling of light curves, where both non-DRW and DRW light curves are equally well-modeled by the DRW method described in Sect. 2, albeit that the former ones result in biased parameters. Because DRW modeling uses an exponential covariance matrix of the signal that translates into the power-law SF slope of 0.5 or the PSD slope of -2 (for $\Delta t<1 \mathrm{yr}$; Kozłowski 2016a), one should make sure that the underlying variability in a light curve is well-described by a DRW process, prior to the DRW modeling. It appears that optical light curves of AGNs seem to have PSD slopes $\alpha \lesssim-2$ or SF slopes $\gamma \gtrsim 0.5$ (Mushotzky et al. 2011; Kasliwal et al. 2015; Simm et al. 2016; Kozłowski 2016a; Caplar et al. 2016), which means that modeling AGN light curves with DRW (having fixed $\alpha \equiv-2$ or $\gamma \equiv 0.5$ ) will produce biased (longer) decorrelation timescales.

Acknowledgements. This work has been supported by the Polish National Science Center grants: OPUS 2014/15/B/ST9/00093 and MAESTRO 2014/14/A/ST9/0012.

\section{References}

Butler, N. R., \& Bloom, J. S. 2011, AJ, 141, 93

Caplar, N., Lilly, S. J., \& Trakhtenbrot, B. 2016, ApJ, submitted [arXiv: 1611.03082]

Collier, S., \& Peterson, B. M. 2001, ApJ, 555, 775

Czerny, B. 2006, ASP Conf. Ser., 360, 2

Dobrzycki, A., Groot, P. J., Macri, L. M., \& Stanek, K. Z. 2002, ApJ, 569, L15

Dobrzycki, A., Macri, L. M., Stanek, K. Z., \& Groot, P. J. 2003a, AJ, 125, 1330 Dobrzycki, A., Stanek, K. Z., Macri, L. M., \& Groot, P. J. 2003b, AJ, 126, 734 Dobrzycki, A., Eyer, L., Stanek, K. Z., \& Macri, L. M. 2005, A\&A, 442, 495 Fausnaugh, M. M., Grier, C. J., Bentz, M. C., et al. 2016, ApJ, submitted [arXiv: 1610.00008 ]

Geha, M., Alcock, C., Allsman, R. A., et al. 2003, AJ, 125, 1

Ivezić, Ž., Smith, J. A., Miknaitis, G., et al. 2007, AJ, 134, 973

Kasliwal, V. P., Vogeley, M. S., \& Richards, G. T. 2015, MNRAS, 451, 4328

Kelly, B. C., Bechtold, J., \& Siemiginowska, A. 2009, ApJ, 698, 895

Kelly, B. C., Becker, A. C., Sobolewska, M., Siemiginowska, A., \& Uttley, P. 2014, ApJ, 788, 33

King, A. 2008, New Astron. Rev., 52, 253

Kozłowski, S. 2016a, ApJ, 826, 118

Kozłowski, S. 2016b, MNRAS, 459, 2787

Kozłowski, S., Kochanek, C. S., Udalski, A., et al. 2010, ApJ, 708, 927

Kozłowski, S., Kochanek, C. S., \& Udalski, A. 2011, ApJS, 194, 22

Kozłowski, S., Kochanek, C. S., Jacyszyn, A. M., et al. 2012, ApJ, 746, 27

Kozłowski, S., Onken, C. A., Kochanek, C. S., et al. 2013, ApJ, 775, 92

MacLeod, C. L., Ivezić, Ž., Kochanek, C. S., et al. 2010, ApJ, 721, 1014

MacLeod, C. L., Brooks, K., Ivezić, Ž., et al. 2011, ApJ, 728, 26

MacLeod, C. L., Ivezić, Ž., Sesar, B., et al. 2012, ApJ, 753, 106

McHardy, I. M., Koerding, E., Knigge, C., Uttley, P., \& Fender, R. P. 2006, Nature, 444, 730

Mushotzky, R. F., Edelson, R., Baumgartner, W., \& Gandhi, P. 2011, ApJ, 743, L12

Press, W. H., Rybicki, G. B., \& Hewitt, J. N. 1992a, ApJ, 385, 404

Press, W. H., Rybicki, G. B., \& Hewitt, J. N. 1992b, ApJ, 385, 416 Ruan, J. J., Anderson, S. F., MacLeod, C. L., et al. 2012, ApJ, 760, 51 Rybicki, G. B., \& Press, W. H. 1995, Phys. Rev. Lett., 74, 1060

Simm, T., Salvato, M., Saglia, R., et al. 2016, A\&A, 585, A129

Skowron, J., Udalski, A., Kozłowski, S., et al. 2016, Acta Astron., 66, 1 Udalski, A., Szymanski, M. K., Soszynski, I., \& Poleski, R. 2008, Acta Astron., 58,69

Udalski, A., Szymański, M. K., \& Szymański, G. 2015, Acta Astron., 65, 1 York, D. G., Adelman, J., Anderson, J. E., Jr., et al. 2000, AJ, 120, 1579 Zu, Y., Kochanek, C. S., \& Peterson, B. M. 2011, ApJ, 735, 80

Zu, Y., Kochanek, C. S., Kozłowski, S., \& Udalski, A. 2013, ApJ, 765, 106 Zu, Y., Kochanek, C. S., Kozłowski, S., \& Peterson, B. M. 2016, ApJ, 819, 122 